\title{
Manometry of the Normal Upper Esophageal Sphincter and its Alterations in Laryngectomy
}

\author{
Richard W. Welch, Kenneth Luckmann, Phillip M. Ricks, Samuel T. Drake, and \\ GeORGE A. Gates, Division of Gastroenterology and Division of \\ Otorhinolaryngology, The University of Texas Health Science Center \\ at San Antonio, San Antonio, Texas 78284
}

\begin{abstract}
A B S T R A C T Rapid pull-through pressure profiles of the normal human upper esophageal sphincter (UES) were simultaneously studied with a conventional three-orifice Honeywell solid-state probe, an eight lumen radially perfused (RP) probe, and a circumferentially sensitive (CS) probe designed to measure UES pressure (UESP) without regard to probe orientation. Pressure curves were digitized and analyzed by computer. The Honeywell probe recorded significantly lower peak pressures than the other two methods, and had wide intrasubject pressure variations (average coefficient of variation, 53\%). In contrast, UESP measured with the CS probe was constant for each subject (mean peak UESP, $121 \mathrm{~mm} \mathrm{Hg}$; average coefficient of variation, 15\%). Anteroposterior RP probe UESP were identical to CS probe pressures. Thus, peak perfused anteroposterior UESP correlates with circumferentially measured sphincter squeeze.
\end{abstract}

Computer programs were written that allowed RP probe pressures to be mapped in three dimensions. Normal three-dimensional maps were characterized by anteroposterior accentuation of peak pressures and also by consistent axial asymmetry with anterior peak pressures occurring $0.8 \pm 0.2 \mathrm{~cm}$ closer to the pharynx.

After defining the normal two- and three-dimensional UESP configuration, patients who had undergone laryngectomy were studied. Peak pressures measured with the $\mathrm{RP}$ probe decreased to $\cong 50 \mathrm{~mm} \mathrm{Hg}$ and radial pressure asymmetry vanished. Like normals, CS probe pressures corresponded to peak RP probe pressures. UES length did not change significantly. Three-dimensional mapping showed that axial asymmetry also vanished. It therefore appears that the anatomic alterations produced by laryngectomy abolishes UESP asymmetry.

Received for publication 11 September 1.978 and in revised form 1.5 January 1979 .

\section{INTRODUCTION}

Upper esophageal sphincter pressure $\left(\mathrm{UESP}^{1}{ }^{1}\right.$ is radially asymmetric (1). This asymmetry makes it difficult to define the normal range of pressures and confounds attempts to detect differences in disease states. It is not known whether this radial array of pressures actually reflects circumferentially variable sphincteral squeeze or whether some of the high pressures recorded with perfused systems are just measurement artifacts. To more precisely define UESP asymmetry and to relate it to conventional methods of measuring UESP, we assessed UESP profiles with two conventional manometric probes and compared these results to a device designed to be circumferentially sensitive to sphincteral squeeze. Data collection and analyses were done by computer, allowing three-dimensional display of pressures and precise definition of the asymmetric features of the normal UESP. We then studied patients with laryngectomy to assess the effect of cricoid cartilage removal on the UESP configuration.

\section{METHODS}

Pressure measuring devices. First, we used a 5.5-mm Diam Honeywell (HW) probe, Honeywell, Inc., Test Instruments Div., Denver, Colo. This probe is the highest fidelity conventional three-orifice probe available. It samples point pressure with three miniature strain gauges mounted in the wall of the probe. These miniature transducers are separated $120^{\circ}$ radially and are mounted $5 \mathrm{~cm}$ apart axially. This arrangement has been traditional for sampling esophageal pressures.

Second, we used a 6 -mm Diam, eight lumen radially perfused (RP) polyvinyl probe whose side holes were all at the same level, thus sampling circumferential pressures at $45^{\circ}$ intervals. Fig. 1 is a cross-section of our RP probe at the level

${ }^{1}$ Abbreviations used in this paper: CS, circumferentially sensitive; $C V$, coefficient of variation, $C V$, mean $\overline{C V}$; $R P$, radially perfused; UES, upper esophageal sphincter; UESP, UES pressure. 


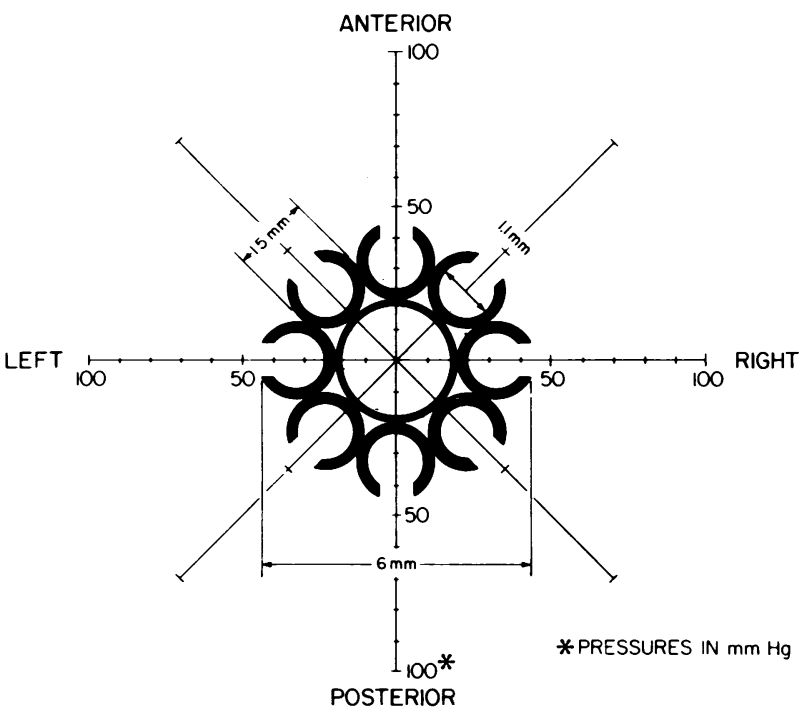

FIGURE 1 Cross-section of the RP probe at the level of the eight side holes. Superimposed is the circular coordinate system we used to map RP pressures.

of the eight orifices and is superimposed on the polar coordinate graphing system we used to map UESP. Orientation of this probe in the UES was confirmed by coloring one of the eight catheters and observing its position as it exited the mouth during pull-through. Fluoroscopic monitoring of a series of pull-throughs done in one volunteer with metallic markings on the catheter confirmed that the probe was sufficiently rigid so that twisting did not occur between the UES and the mouth. The eight lumen catheter was perfused with an Arndorfer hydraulic-pneumatic perfusion device at $1 \mathrm{ml} / \mathrm{min}$ per channel. Before the experiment, cross-clamping each channel produced a rate of rise $>300 \mathrm{~mm} \mathrm{Hg} / \mathrm{s}$.

Third, we used a 6-mm Diam motility probe specially designed for us by the Konigsberg Instruments Co., Inc., Pasadena, Calif. (Fig. 2). This probe is a glycerine-filled silastic torus mounted $10 \mathrm{~cm}$ from the end of an $80-\mathrm{cm}$ silastic tube. The torus surrounds a single miniature titanium strain gauge in contact with the glycerine. This device, which we have called a circumferentially sensitive (CS) probe, provides a measure of circumferential sphincter squeeze by hydrauli-

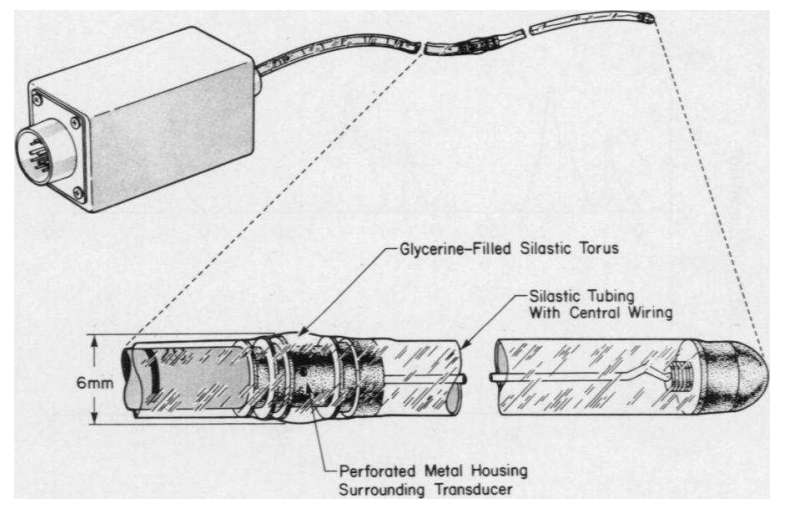

FIgure 2 Diagram of the CS probe. The slight bulge identifies the glycerine-filled chamber. cally transmitting pressures from around the circumference of the sphincter to the strain gauge. The device was found to have an in vitro frequency response of $80 \mathrm{~Hz}$. A similar instrument has been reported by Kaye et al. (2). All three instruments were calibrated with a mercury sphygmomanometer before each experiment. In addition, the CS probe was calibrated weekly with a micrometer by indenting its surface 0.04 in. The pressure obtained by this maneuver was 48-52 $\mathrm{mm} \mathrm{Hg}$. Thus, small indentations of the torus cause increases in recorded pressures, confirming the device's low compliance. The pressures produced by this calibration decreased suddenly at the end of $1 \mathrm{yr}$. It was found that glycerine had leaked from the torus and needed to be refilled.

Two time-synchronized Narco physiographs recorded up to 10 channels of pressure data simultaneously.

Subjects. This study was approved by the Human Use Committee of The University of Texas Health Science Center at San Antonio. 26 normal subjects (mean age, 32; range, 26-65) had their UESP measured with both the conventional three-transducer HW probe and the CS probe. 13 of these same subjects (mean age, 35; range, 26-65) also had their UESP measured with the RP probe. The normal subjects were drawn from two groups. First, we recruited 14 medical students and faculty. These subjects had no history of dyspepsia or other gastrointestinal diseases and did not undergo any further diagnostic studies. Next, we studied 12 patients who underwent esophageal motility as part of a diagnostic work-up for chest pain. These subjects did not complain of dysphagia, and did not have esophageal disease as determined by upper gastrointestinal series and esophagram, endoscopy (usually with biopsy), and conventional esophageal motility. These patients were felt to have either functional symptoms or a disease unrelated to the esophagus. They were included in the normal group to obtain a more precise statistical description of the performance of the $\mathrm{HW}$ and CS probes. We then studied 11 patients (mean age, 55; range, 42-74) who had undergone total laryngectomy 6 mo to $10 \mathrm{yr}$ previously for localized carcinoma of the larynx. Total laryngectomy includes surgical excision of the larynx, strap muscles, and hyoid bone, with a three-layer reapposition of the muscle in the sphincteric region. Thus, UESP configuration in these patients was not influenced by the anteriorly situated cricoid cartilage or other laryngeal structures.

Methods. The method for measuring UESP was a modification of the rapid pull-through technique popularized by Dodds et al. (3). The rapid pull-through technique is very desirable in the UES, as perfusate does not have time to accumulate in the hypopharynx to cause coughing. Because one goal was to measure sphincter length, the probe was withdrawn through the UES with a motor-pulley device at exactly $0.5 \mathrm{~cm} / \mathrm{s}$. Because all data channels were time synchronized and the paper speed was matched at $0.5 \mathrm{~cm} / \mathrm{s}$, length on the graph paper represented sphincter length. Each of the three probes was withdrawn through the upper sphincter six times in succession, and then the next probe was immediately inserted and the procedure was repeated. Six measurements were chosen because statistical analysis of up to 10 rapid pull-throughs in the first seven subjects indicated no significant change in the mean pressure or standard deviation beyond six determinations. The patient's sphincter pressure was measured orally in the right lateral decubitus position with the breath held in mid-inspiration. Particular attention was paid to avoid deep inspiration or the Valsalva maneuver and to always position the patient's neck in comfortable extension. Preliminary studies showed posteriorly measured UESP to vary widely with the neck flexed. Restudy of two subjects in the supine and left lateral decubitus positions confirmed that pressure profiles were not altered by positional changes. 
Data analysis. Sphincter pressures were recorded as millimeters of mercury above esophageal base-line pressure. Because esophageal pressure is less than atmospheric, a precise definition of the oral end of the upper esophageal high pressure zone has to take this pressure difference into account. At $50 \%$ vital capacity (mid-inspiration), Milic-Emili et al. (4) have shown this pressure difference to average $10 \mathrm{~mm} \mathrm{Hg}$. We therefore defined $10 \mathrm{~mm} \mathrm{Hg}$ above esophageal base-line as the oral end of the UES. UESP generated with the CS and RP probes were analyzed by computer to obtain precise, unbiased measurements of peak UESP, length, and area under CS pressure profile. UESP measurements with the HW probe were so variable that further computer analysis of the data was not done. Our computer technique involved tracing the withdrawal profile of the UES with a Tektronix 4010-1 digitizer (Tektronix, Inc., Beaverton, Oreg.). This device, which is coupled to a PDP $11 / 50$ computer (Digital Equipment Corp., Marlboro, Mass.), is an electromagnetic stylus that is moved over a grid containing $10^{6}$ contact points. The pressure curve is thereby transformed into a series of closely spaced but discrete points that are analyzed by computer to provide an unbiased measure of peak pressure, length, and area under the sphincteric pressure curve. The technique for three-dimensional mapping also involves digitizing the sphincter profiles. The eight curves obtained with the RP method are digitized and pressure is sampled at $0.1-\mathrm{cm}$ intervals. The computer is then programmed to arrange these points in three-dimensional space and connect the time-synchronous pressures with a smooth curve. This curve-fitting involved mathematical modeling with spline function analysis (5). The "hidden line" software program allowing three-dimensional graphic display was developed by the Computer Center at The University of Texas Health Science Center at San Antonio. This method allows simultaneous visual display of the radial and axial shape of the UES. Subsequent to this study, we have begun entering data directly into the computer from the pressure transducers by means of analog to digital converters, thus bypassing the recorder and the need for digitization.

Statistical analysis was incorporated into the final computer program. The standard error of the mean was used to describe the distribution of all values around the mean. Because all UES measurements were done the same number of times with each device, a paired Student's $t$ test was used to describe statistical differences between the HW, CS, and RP probes. An unpaired Student's $t$ test was used to compare UESP, length, and area in the laryngectomy patients with the normals. The coefficient of variation was used to describe the measurement precision of each device. A mean coefficient of variation was used to describe average overall variations with the HW and CS probes and each port of the RP probe. The statistical significance of differences between the instruments' variabilities were assessed by a two-way analysis of variance.

\section{RESULTS}

Normal subjects. Peak upper sphincter pressure was first measured with the HW and CS probes in the 26 normal subjects. The six successive pull-throughs with the HW probe showed large variations in peak pressure. The coefficient of variation $(\mathrm{CV})$ for each subject ranged from 22 to $89 \%$ with a mean coefficient of variation $(\overline{\mathrm{CV}})$ for all 26 subjects of $53 \%$. Length measurements with $\mathrm{HW}$ were also unreliable (average length, $2.6 \mathrm{~cm} ; \overline{\mathrm{CV}}, 64 \%$ ). This marked $\mathrm{HW}$ probe vari- ability precluded meaningful computer assessment. UESP measured by the CS probe was far more precise with only a $15 \% \overline{\mathrm{CV}}$ measured for all subjects. The variability with the CS probe was significantly less than with the HW probe $(P<0.01)$. Mean peak UESP for all subjects was also significantly higher with the CS device $(121 \mathrm{~mm} \mathrm{Hg})$ than with the $\mathrm{HW}$ probe $(76 \mathrm{~mm}$ HG, $P<0.05)$.

The contour of the CS probe pressure profiles, but not the HW probe, was characteristic and reproducible for each subject, and was either unimodal or bimodal. Fig. 3 is an example of this uniformity in one subject. Because of this reproducible UESP profile, meaningful integration of the area under the curve was possible. This area, whose units are millimeters of mercury times centimeters, represents the total pressure barrier of the UES. In the 26 normal subjects, computerized UES length was $3.1 \pm 0.2 \mathrm{~cm}$ and UES area was $160 \pm 24 \mathrm{~mm}$ $\mathrm{Hg}-\mathrm{cm}$.

Upper sphincter pressure was also measured in 13 of these normal subjects with the eight lumen RP probe. We were therefore able to directly compare UESP measured with the RP probe with that measured with the CS probe. Fig. 4 shows comparison of the eight individual profiles on a single RP probe pull-through with that obtained immediately afterwards with the CS probe. Pressure fluctuations seen with the CS device correspond to changes in pressure seen in each of the ports of the RP probe. The CS probe's curves therefore appears to represent a composite profile of all eight $\mathrm{RP}$ probe withdrawal profiles, and allows an overall assessment of the pressure barrier of the UES not possible by analyzing any one of the eight individual RP profiles.

Fig. 5 compares the pressures obtained with the CS probe to those obtained with the RP probe in the 13 subjects. With the RP probe, pressures are maximum in the anteroposterior plane and decrease rapidly in the lateral directions. For the six pull-throughs for each subject, the eight lumen RP reproducibly recorded

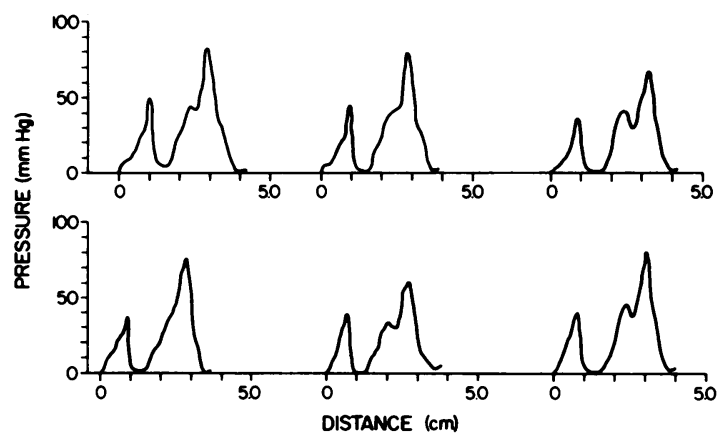

FIGURE 3 Six consecutive pressure profiles of the UES measured by the CS probe. Notice the uniformity in overall contour, peak height, and length for each of the pull-throughs. 


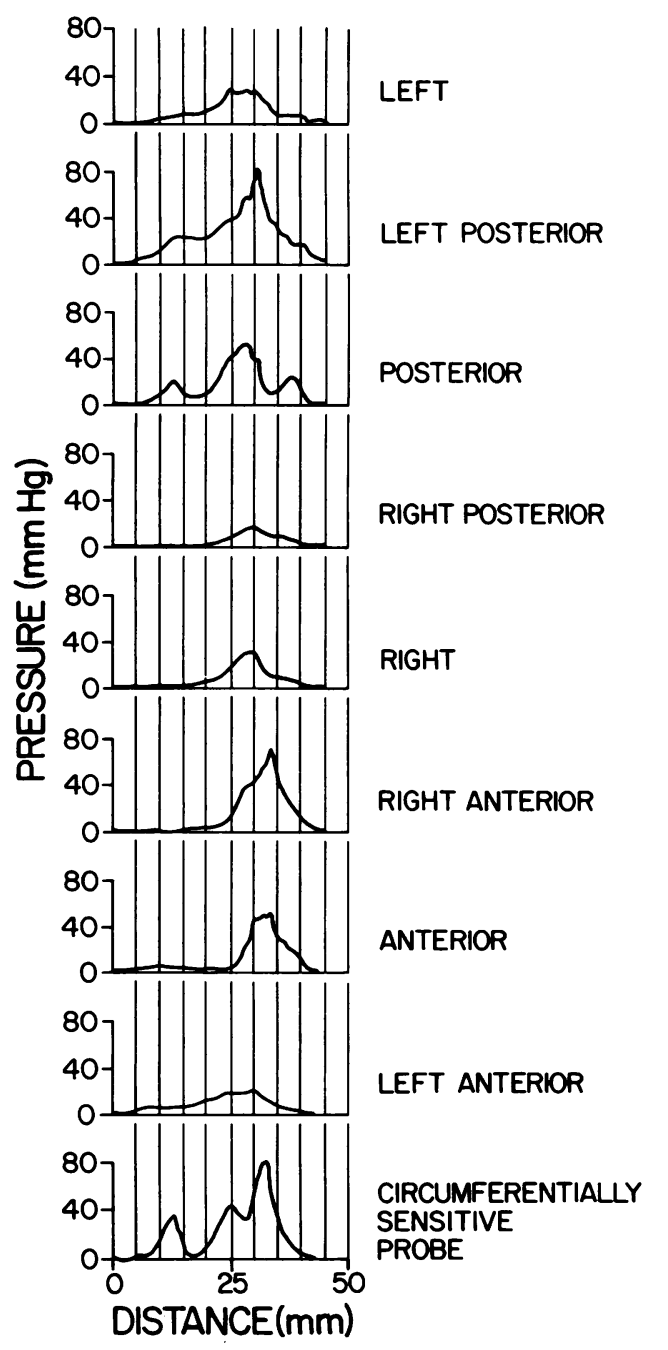

FIGURE 4 Withdrawal profiles of the eight RP pressures compared to pressures measured by the CS probe. Pressure changes occurring in one or more RP probe channels are reflected in the CS probe pressure profile.

peak pressures, with the $\overline{\mathrm{CV}}$ for each port varying from 17 to $23 \%$. In Fig. 5, the peak CS probe UESP is represented as a circle and corresponds exactly to the mean peak RP probe UESP in the anteroposterior plane.

The RP probe data for each subject were then expanded into three dimensions with the aid of the computer. The eight individual RP profiles were digitized for all six pull-throughs in each of the 13 subjects. Each pull-through produced a three-dimensional pressure map of both the axial and radial asymmetry. Fig. 6 is a three-dimensional map of eight individual RP probe profiles obtained in one pull-through and is the same pull-through shown in Fig. 4. To reveal the hidden side of the sphincter, the graphic representation was rotated $180^{\circ}$ on the sphincter's axis as is shown in the lower panel of Fig. 6. Notice the marked anteroposterior ac-

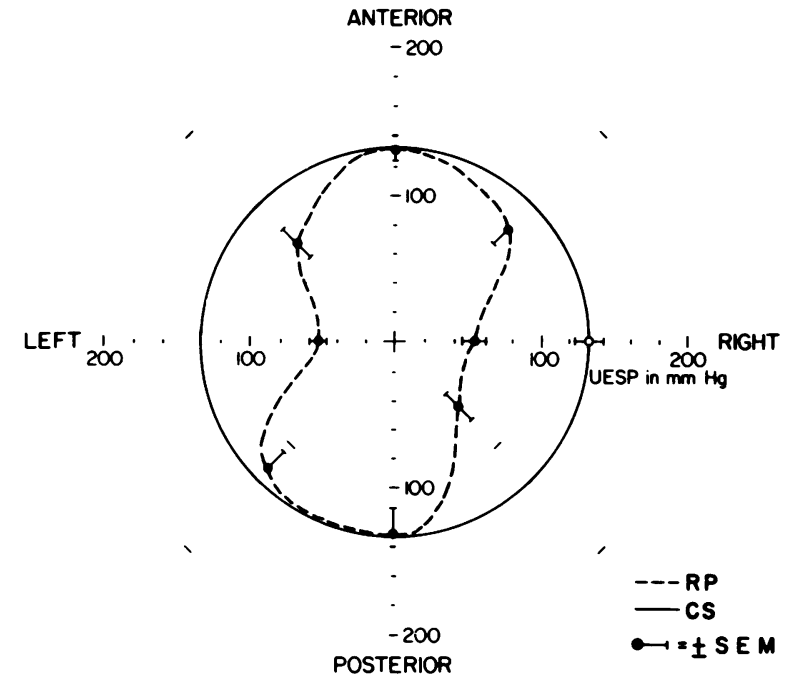

FIGURE 5 A comparison of RP UESP (-- - ) to pressures measured with the CS probe ( - ).

centuation of pressures in the region of peak pressure. In all subjects studied with the computerized threedimensional modeling, this prominent pressure bulge was $\cong 1 \mathrm{~cm}$ in width and occurred near the upper boundary of the UES. Furthermore, in all 13 subjects studied, the anterior peak of this bulge was closer to the pharynx than the posterior peak. Statistical comparison of the peak posterior pressure vs. the peak anterior pressure revealed that the anterior peak was located $0.8 \pm 0.2 \mathrm{~cm}$ nearer the pharynx compared to the posterior peak, a highly significant difference $(P<0.001)$. Thus, axial, as well as radial, asymmetry is a consistent feature of the human UES.

Patients who underwent laryngectomy. Computer analysis of the CS and RP probe data from the 11 patients who had undergone total laryngectomy was done

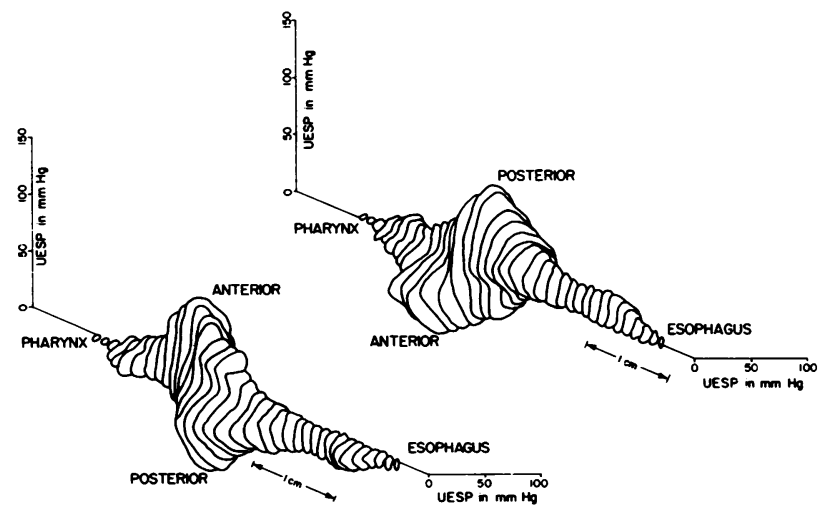

FIGURE 6 Three-dimensional pressure profile of the normal UES. Notice the anteroposterior accentuation of pressure and the axial asymmetry with an orad shift of anterior pressures. In the lower panel, the graphic representation was rotated $180^{\circ}$ to reveal the hidden side of the sphincter. 
to assess the effect of total laryngectomy on UESP asymmetry. Peak CS probe UESP in these patients was decreased to $51 \pm 8 \mathrm{~mm} \mathrm{Hg}$ as compared to $121 \pm 14$ in normals $(P<0.01)$. Sphincter length was $3.5 \pm 0.6 \mathrm{~mm}$ $\mathrm{Hg}$ compared to $3.1 \pm 0.2$ in normals $(P<0.2)$, and pressure area was reduced to $80 \mathrm{~mm} \mathrm{Hg}$ compared to $160 \pm 24$ in normals $(P<0.01)$. Moreover, peak RP probe pressure maps were nearly circular, varying from 45 to $52 \mathrm{~mm} \mathrm{Hg}$ in the eight directions, and corresponded closely to $\mathrm{CS}$ probe pressure of 51 . Furthermore, these RP probe pressures were nearly identical to the lateral RP pressures found in the normals (Fig. 7). In addition, three-dimensional mapping failed to reveal any consistent axial asymmetry. Fig. 8 is a representative three-dimensional map from one subject, and demonstrates the loss of asymmetry.

\section{DISCUSSION}

Conventional nonoriented methods for measuring UESP, such as the HW probe, are unreliable. Lower pressures (HW probe, $76 \mathrm{~mm} \mathrm{Hg}$ vs. CS probe, $121 \mathrm{~mm}$ $\mathrm{Hg}$ ) with wide variations for each pull-through (HW probes $\overline{C V}, 53 \%$ vs. CS probe's $\overline{C V}, 15 \%$ ) occur because exact anteroposterior placement of the HW-recording port is difficult. Although this result might have been predicted, we felt careful documentation of the magnitude of this error was necessary to definitely invalidate all prior nonoriented studies. Precise sphincteric radial orientation or a circumferentially sensitive probe is a requisite for studies of the UES.

The $15 \%$ CV obtained when UESP was measured with the CS device means that the UES squeezed with a relatively constant pressure over the duration of the study. This constancy should allow detection of modest

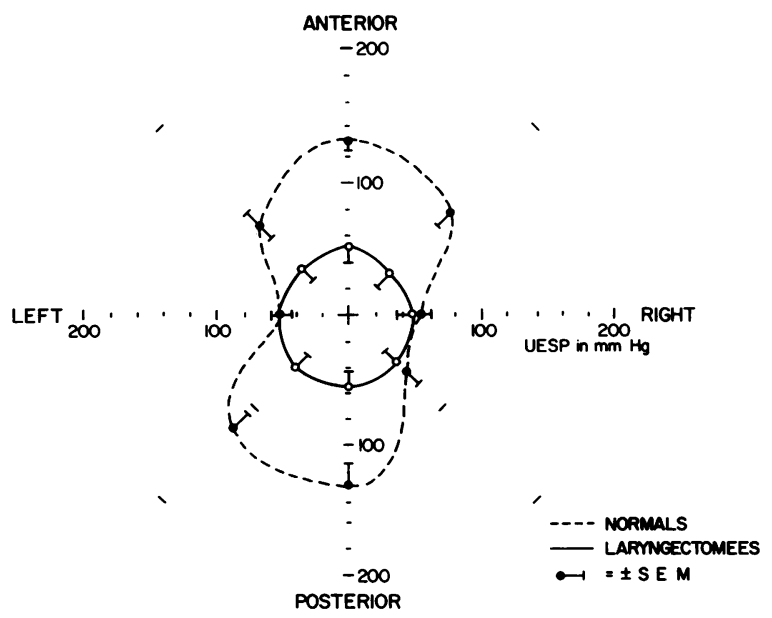

FIGURE 7 Comparison of UES RP pressures in 13 normal subjects $(---)$ to those of 11 laryngectomees (-). Notice the circular pressures in the laryngectomees and the identical lateral pressures in the two groups.

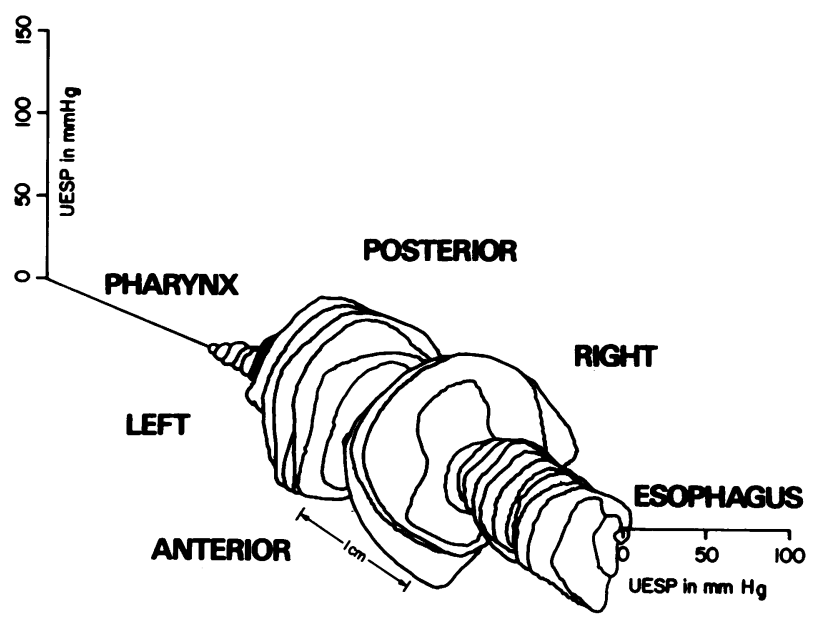

FIGURE 8 Three-dimensional pressure profile of the UES in a representative laryngectomee. Notice the lack of radial and axial asymmetry.

changes in sphincteric tone in short-term studies. The possibility still exists that over longer time intervals cyclic changes in sphincteric pressures might exist. The average peak UESP of $\cong 120 \mathrm{~mm} \mathrm{Hg}$ found in this study is undoubtedly higher than the UES squeezes in the absence of a catheter, as this high pressure would cause the mucosal surface to become ischemic. Thus, the "normal" values reported in this study apply only for a 6-mm catheter.

With the CS probe, the shape of the UESP withdrawal profiles appeared to be reproducible on each of the six pull-throughs for each subject studied and were composites of the eight $45^{\circ}$ radially separated $\mathrm{RP}$ profiles (Figs. 3 and 4). This finding allowed us to calculate the area under the CS probe's withdrawal profile. This area represents the total pressure barrier of the UES and takes into account the length of the sphincter as well as the peak pressure obtained. Meaningful calculation of the total pressure barrier with the RP data is much more difficult, because each of the eight RP profiles obtained on a single pull-through vary greatly in length, peak pressure, contour, and axial orientation. The concept that a long pressure barrier more effectively resists flow is not new. Alexander et al. (6) have mathematically documented the clinical observation that too narrow a blood pressure cuff causes a falsely elevated reading. That is, the arterial opening pressure depends upon the length of the occluded segment; if the cuff is too short, more pressure is necessary to resist flow. In general, elastic pressure barriers shorter than the diameter of the conduit are most likely subject to this phenomenon. Analogously, reflux of esophageal contents into the hypopharynx might be more effectively prevented with a longer high pressure zone than a shorter one, even if peak pressures were identical.

Unlike the RP probe, the CS probe does not depend 
upon the idiosyncrasies of fluid flow through elastic tubes. Perfused manometry measures the opening pressure of a catheter orifice occluded by the sphincter and may depend upon the compliance of structures surrounding the opening. The UES is anatomically complex; its mucosa is backed partially by muscle and partially by cartilage. It has not been proven that all of the perfused opening pressures actually reflect sphincteric squeeze. For example, when the catheter orifice is resting against the unyielding cricoid cartilage one might expect a higher sphincter pressure than when resting upon muscle. The ability to simultaneously compare conventional perfused pressures (RP probe) with an independent measure of sphincter pressure (CS probe) helps evaluate the wide range of pressures found in the UES. The CS-RP probe comparison confirms that maximum recorded perfused pressures do reflect true sphincteric squeeze and are not measurement artifacts. The CS probe appears to be a rapid, convenient method for recording maximum sphincter pressure without the need for radial orientation.

Insight into the cause of the radial pressure asymmetry of the UES can be gained by inspecting the RP and CS probe data in laryngectomees. All of these patients had the usual total laryngectomy with perioperative irradiation for localized laryngeal carcinoma. This operation results in excision of the laryngeal structures including the cricoid cartilage from the muscle layer in the region of the UES with a three-layer, midline reapproximation of the incised muscle. Surgery may disrupt some, but not all of the nervous supply to the UES. When the UES loses the anterior situated larynx and cricoid cartilage, the radial asymmetry vanishes, pressure and area decrease, and the pressure map becomes circular. Sphincter length is not statistically different than the normals. Furthermore, the mean lateral RP pressures in normals are very similar to the RP pressures found in the laryngectomy patients.

UESP axial asymmetry also disappears when the cricoid cartilage and other laryngeal structures are removed. There are several possible explanations for the disappearance of UES asymmetry postlaryngectomy, such as radiation injury, surgical denervation, narrowing or destruction of the sphincter. Another more reasonable hypothesis is that the sphincteric musculature, which attaches anteriorly to the cricoid cartilage, functions like the jaws of a vise. The anterior moveable jaw would be the cricoid cartilage and other laryngeal structures and the posterior, fixed jaw would be the sphincteric muscle applied to the cervical spine. The UES axial asymmetry implies that the "jaws" of the UES do not exactly oppose each other but rather are partially offset. Asoh and Goyal (7) have recently demonstrated UES axial asymmetry in the opossum. Interestingly, in this species the posterior, not the anterior, pressure peak is closer to the pharynx.
Sophisticated computer analysis of RP motility tracings has allowed us to topographically map the complex asymmetric pressures of the UES in three dimensions. The eight simultaneous and seemingly discordant profiles obtained with one pull-through of the RP system have been shown to be part of a series of harmonious and smooth changes in pressure that are strikingly demonstrated by the three-dimensional computer mapping. This mapping technique graphically displays the radial and axial asymmetry of the human UES. We believe computer processing and displaying of the total family of pressures obtained in physiologic high pressure zones is an excellent way to study the sphincter's normal and pathological behavior. It appears premature to accept any single value for sphincter pressure, such as the CS probe produces, without extensive comparison with all the sphincteric pressures generated from the three-dimensional technique.

Our computer techniques may have additional applications in the measurement of other biologic systems under pressure. Not only can three-dimensional pressure profiles be generated, but by using analog to digital convertors direct on line computer processing of pressure data is possible.

\section{ACKNOWLEDGMENTS}

The authors wish to thank Cindy Lozano for her typing of the manuscript and Bernie Schelper, medical technician, for her technical assistance.

This investigation was supported in part by research grants 7R01AM25609-01, 5501RR05654-07, subgrant S07RR05654 from the National Institutes of Health, and R18CA18629 from the National Cancer Institute.

\section{REFERENCES}

1. Winans, C. S. 1972. The pharyngoesophageal closure mechanism: a manometric study. Gastroenterology. 63: 768-777.

2. Kaye, M. D., J. P. Showalter, K. C. Rock, and E. Johnson. 1973. A circumferentially-sensitive miniature transducer for study of human esophageal motility. Gastroenterology. 64: 752. (Abstr.)

3. Dodds, W. J., W. J. Hogan, J. J. Stef, W. N. Miller, S. B. Lydon, and R. C. Arndorfer. 1975. A rapid pull-through technique for measuring lower esophageal sphincter pressure. Gastroenterology. 68: 437-443.

4. Milic-Emil, J., J. Mead, J. M. Turner, and E. M. Glauser. 1964. Improved technique for estimating pleural pressure from esophageal balloon. J. Appl. Physiol. 19: 207-211.

5. Cline, A. K. 1974. Scalar and planar-valued curve fitting using splines under tension. In Communications of the Association for Computing Machinery. R. Willoughby, editor. 17: 218-221.

6. Alexander, H., M. L. Cohen, and L. Steinfeld. 1977. Criteria in the choice of an occluding cuff for the indirect measurement of blood pressure. Med. Biol. Eng. Comput. 15: 2-10.

7. Asoh, R., and R. K. Goyal. 1978. Manometry and electromyography of the upper esophageal sphincter in the opossum. Gastroenterology. 74: 514-520. 